\title{
High resolution spectroscopy of HgMn stars: a time of surprises
}

\author{
S. Hubrig ${ }^{1}$, C.R. Cowley $^{2}$, F. González ${ }^{3}$, F. Castelli ${ }^{4}$ \\ 1 European Southern Observatory, Casilla 19001, Santiago, Chile \\ shubrig@eso.org, \\ 2 University of Michigan, Ann Arbor, MI 48109-1042, USA cowley@umich.edu \\ 3 Complejo Astronómico El Leoncito, Casilla 467, 5400 San Juan, Argentina \\ fgonzalez@casleo.gov.ar, \\ 4 Osservatorio Astronomico di Trieste, Trieste, Italy castelli@ts.astro.it
}

The origin of the abundance anomalies observed in late B-type stars with $\mathrm{HgMn}$ peculiarity is still poorly understood. Observationally, these stars are characterized by low rotational velocities and weak or non-detectable magnetic fields. The most distinctive features of their atmospheres are an extreme overabundance of $\mathrm{Hg}$ (up to 6 dex) and/or Mn (up to 3 dex) and a deficiency of He. Anomalous isotopic abundances have been reported in the past for the elements $\mathrm{Hg}, \mathrm{Pt}$ and $\mathrm{Tl}$. Observational evidence for large isotopic shifts in the infrared triplet of Ca II was presented in the last two years (Castelli \& Hubrig 2004 [1], Cowley \& Hubrig 2005 [2, Cowley et al., these proceedings). Shifts of up to $+0.2 \AA$ were found in a number of $\mathrm{HgMn}$ and magnetic Ap stars indicating the dominant isotope is the terrestrially rare ${ }^{48} \mathrm{Ca}$.

As more than 2/3 of the $\mathrm{HgMn}$ stars are known to belong to spectroscopic binaries (Hubrig \& Mathys 1995 [3]), the variation of spectral lines observed in any HgMn star is usually explained to be due to the orbital motion of the companion. Here we present the results of a high spectral resolution study of a few spectroscopic binaries with HgMn primary stars. We detect for the first time in the spectra of HgMn stars that for many elements the line profiles are variable over the rotation period (Hubrig et al. 2006 [4). The strongest profile variations are found for the elements $\mathrm{Pt}, \mathrm{Hg}, \mathrm{Sr}, \mathrm{Y}, \mathrm{Zr}, \mathrm{Mn}, \mathrm{Ga}, \mathrm{He}$ and $\mathrm{Nd}$. The slight variability of $\mathrm{He}$ and $\mathrm{Y}$ is also confirmed by the study of high resolution spectra of another HgMn star, $\alpha$ And.

In Fig. 1 we show the behavior of the line profile of $\mathrm{Hg}$ II $\lambda 3983.9$ in the spectra of AR Aur at different rotation phases. Our preliminary modelling of abundance distributions of the elements $\mathrm{Sr}$ and $\mathrm{Y}$ over the stellar surface suggests that these elements are very likely concentrated in a fractured ring along the rotational equator (Hubrig et al. 2006 [4). In Fig. 2 we present recent FEROS observations of variable line profiles of Y II $\lambda 3982.5$ in the spectra of the HgMn star HD 11753 and of Y II/Hg II lines in the spectra of the HgMn double-lined spectroscopic binary HD 27376.

The discovery of an inhomogeneous distribution of various elements in the atmospheres of $\mathrm{HgMn}$ stars challenges our understanding of the nature of HgMn stars. We believe that factors as the presence of a weak tangled 


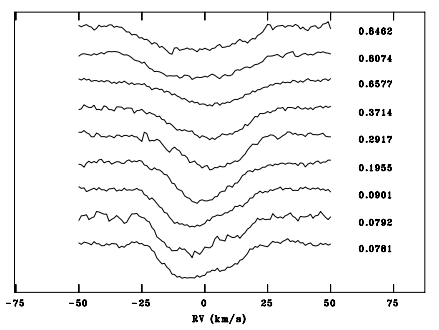

Fig. 1. Variations of the line profile of $\mathrm{Hg}$ II $\lambda 3983.9$ in the spectra of AR Aur phased with the rotation period $\mathrm{P}=4.13$ days.
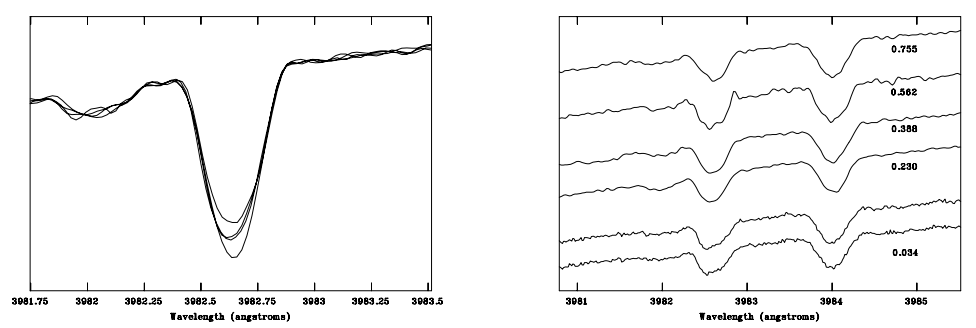

Fig. 2. Left: Profile variations of the Y II $\lambda 3982.5 \AA$ line in the spectra of HD 11753 at various rotation phases. Right: Profile variations of the Y II $\lambda 3982.5$ and $\mathrm{Hg}$ II $\lambda 3983.9$ lines in the spectra of HD 32964 over the rotation period.

magnetic field, tidal distortion, or the reflection effect can play a role in the development of anomalies in HgMn stars. Although diffusion due to gravitational settling and radiative levitation remain the most popular explanation for the HgMn star abundances, the selective accretion of interstellar material during the pre-main-sequence phase by $\mathrm{HgMn}$ binary systems seems to be a promising possibility for explaining some surface anomalies, given the presence of magnetic fields. Probably, all these mechanisms have to be taken into account in future studies of these stars.

\section{References}

1. Castelli F., Hubrig S. 2004, A\&A 421, L1

2. Cowley C.R., Hubrig S. 2005, A\&A 432, L21

3. Hubrig S., Mathys G. 1995, ComAp 18, 167

4. Hubrig S., González J.F., Savanov I., et al. 2006, MNRAS 371, 1953 